\title{
La dispute identitaire espagnole et catalane à propos des corridas
}

\author{
Ludivine Thouverez ${ }^{1}$ \\ Cristina Perales García ${ }^{2}$
}

\begin{abstract}
[Résumé] L'interdiction de la corrida, votée par le parlement autonome de Catalogne en 2010, a été à l'origine d'une grande controverse en Espagne. Cet article analyse la couverture de l'événement dans quatre journaux issus du centre et de la périphérie, dans le but de déterminer s'ils se sont emparés du débat pour conforter leur discours identitaire respectif, contribuant ainsi à l'escalade du conflit. L'approche de l'Analyse critique du Discours permettra de révéler comment les croyances idéologiques se (re)produisent dans les textes et comment les quotidiens participent à la construction des identités catalane et espagnole, à travers l'émission d'arguments favorables ou contraires aux spectacles taurins.
\end{abstract}

Mots-clés : conflit politique, identité, discours, presse, journalisme, Espagne

[Abstract] The ban on bullfighting, which was passed by the Parliament in Catalonia in 2010, raised much controversy in Spain. This research analyses the media coverage of the event, by four newspapers from the centre and periphery, and looks at whether they use the debate as a pretext for reaffirming their position with regard to identity and whether they contribute to the polarization of the conflict. A Critical Discourse Analysis approach is used in order to uncover how ideological beliefs are spread and how the newspapers help to establish Spanish and Catalan identity, setting out the arguments for and against bullfighting.

Keywords: political conflict, identity, discourse, journalism, press, Spain

\section{Introduction}

En juillet 2010, la décision du parlement de Catalogne d'interdire la corrida sur son territoire a déclenché une grande controverse en Espagne. Le débat, initialement centré sur la question de la protection des animaux, s'est transformé rapidement en querelle identitaire. Partant du postulat que les médias sont des acteurs politiques et que leurs discours influencent l'interprétation et l'évolution des conflits, cet article compare la représentation de l'événement dans quatre journaux de référence, issus du 
centre (Madrid) et de la périphérie (Catalogne) ${ }^{3}$, dans le but de déterminer si l'abolition de la corrida les conduit à réaffirmer leur propre discours identitaire, contribuant ainsi à la polarisation de la querelle. L'approche de l'Analyse critique du Discours permettra de révéler comment les croyances idéologiques se (re)produisent dans les textes et comment les quotidiens participent à la construction des identités catalane et espagnole, à travers l'émission d'arguments favorables ou contraires aux spectacles taurins.

\section{Contexte d'une abolition}

Si l'organisation de jeux taurins est attestée depuis l'Antiquité dans les pays du bassin méditerranéen, c'est en Espagne que s'est développée la forme actuelle de la corrida qui inclut la mise à mort d'un taureau par un matador - à partir du XVIIIe siècle et a perduré jusqu'à nos jours. L'engouement pour cette pratique est tel que le taureau est devenu un symbole de l'identité culturelle espagnole. Un mouvement social antitaurin s'est pourtant développé à la fin du XXe siècle. La communauté autonome des Canaries fut la première à abolir la corrida en 1991, officiellement en raison du manque d'intérêt qu'elle suscitait. Les combats de coqs, considérés comme une tradition culturelle de l'archipel, échappèrent à l'interdiction. Le mouvement abolitionniste s'étendit ensuite au reste de l'Espagne. Barcelone se déclara "ville anti-taurine » en 2004, mais c'est à la suite d'une pétition recueillant 180.000 signatures que le parlement de Catalogne décida d'interdire la corrida le 30 juillet 2010, sur l'ensemble de la région autonome. Il autorisa néanmoins le maintien des correbous, une tradition régionale qui consiste à fixer des boules de feu sur les cornes des taureaux.

Si les lois canarienne et catalane présentent plusieurs similitudes, les réactions à leur adoption furent totalement différentes : la première fut globalement respectée tandis que l'interdiction de la corrida en Catalogne entraîna une controverse qui relégua la cause animale à un plan secondaire, pour laisser place à des querelles identitaires. En effet, le débat s'inscrit dans un contexte de tensions politiques entre la Catalogne et l'Espagne: entre 2009 et 2011, la moitié des villes catalanes organisent des consultations populaires au cours desquelles $92,2 \%$ des votants se déclarent favorables à l'autodétermination. En juin 2010, le Tribunal constitutionnel annule plusieurs articles du nouveau statut d'autonomie catalan. Le mois suivant, un million de

3 Selon A. Pérez-Agote (1993), le manque d'adéquation entre l'étendue territoriale de l'État espagnol et l'étendue du sentiment d'appartenance nationale a déterminé la naissance de nationalismes périphériques en Catalogne, Galice et au Pays basque.

4 Charaudeau (2006) définit l'identité comme "un ensemble de traits identitaires plus ou moins stables, plus ou moins mouvants, qui participent tous des imaginaires qui circulent à l'intérieur des groupes sociaux ». 
manifestants répliquent au cri de "Nous sommes une nation : c'est à nous de décider», à Barcelone. La succession de ces événements influence la perception de l'abolition, laquelle ne tarde pas à être associée à une autre question: celle de l'intégration de la Catalogne, et donc des territoires périphériques, au sein de l'État espagnol.

\section{Identités, conflits et discours}

L'identité n'est pas une réalité objective mais une représentation mentale que l'individu se fait de lui-même et du groupe. Si plusieurs individus estiment faire partie d'un même groupe, on peut alors parler de sentiment d'appartenance partagée, et donc, d'identité collective. La construction identitaire passe par un double processus : en tant qu'être unique, l'individu prend conscience de sa différence à l'égard des autres individus ; et en tant que membre d'un groupe, il s'identifie à certains comportements, valeurs et traditions qu'il oppose à ceux des autres groupes. Voilà pourquoi Charaudeau (2006) affirme: «l'appartenance à un groupe, c'est d'abord la nonappartenance à un autre groupe, et la quête du groupe, en tant qu'entité collective, c'est également la quête du non-autre».

Les identités nationales, qui constituent une forme d'appartenance à une identité collective, résultent d'une action d'acteurs politiques, de conditions socioéconomiques particulières, ainsi que de la production de discours motivant la croyance en une histoire commune et l'adhésion du public à cette représentation collective du «nous» (Maíz 1997: 5). Le discours est donc au cœur de la construction identitaire (Louw 2005) et les médias, en leur qualité d'espace public de représentation, participent à la configuration des identités collectives, tout comme aux conflits, auxquels sont liées les notions d'altérité et d'identité.

Comme le remarquent Wodak et Meyer (2003: 113), les discours identitaires peuvent créer ou attiser des tensions entre groupes. Dans la mesure où les médias interprètent les conflits et que cette activité n'est jamais neutre - le choix d'accorder de la visibilité à une querelle, de l'inscrire dans telle ou telle rubrique ou d'employer un langage spécifique pour décrire ses acteurs influence la perception que le public aura de celle-ci - il est possible d'affirmer, en accord avec Borrat (1989), qu'ils sont acteurs des conflits.

L’Analyse critique du Discours (ACD), qui conçoit le langage comme une pratique sociale s'articulant autour de trois paramètres (l'identité personnelle, les représentations du monde et les relations entre les acteurs participant à l'acte de communication), porte un intérêt particulier aux conflits sociaux et à leur manifestation dans les discours institutionnels, politiques et médiatiques. Considérant que tout discours est un objet produit dans un espace et un temps donnés et que les structures de domination de la société se trouvent légitimées au moyen de l'idéologie, l'ACD se propose d'étudier : 
Comment l'inégalité sociale s'exprime, se manifeste, se construit et se trouve légitimée dans l'emploi du langage (c'est-à-dire dans le discours). La plupart des analystes du discours accepteraient par conséquent la définition d'Habermas, pour qui 'le langage est aussi un instrument de domination et une force sociale'. II sert à rendre légitime les relations de pouvoir organisé. En ce sens, il est aussi idéologique (Wodak et Meyer 2003 : 19).

De cette manière, l'ACD ne se limite pas à une étude descriptive des textes : elle interprète et explique leurs caractéristiques en fonction du contexte socio-discursif. Étant donné que les journaux produisent des récits qui ne seraient pas fidèles à la réalité mais seraient plutôt conformes à leur ligne éditoriale et aux attentes de leur public, cette approche sociale du langage offre un cadre particulièrement adapté à l'étude des discours de presse.

\section{Méthodologie}

Notre corpus est constitué de 148 articles d'information et d'opinion publiés entre le 25 et le 31 juillet 2010, c'est-à-dire durant la période correspondant au débat et au vote parlementaires autour de l'interdiction de la corrida en Catalogne, dans quatre journaux représentatifs de deux contextes : El País et El Mundo pour le centre (Madrid) ; La Vanguardia et El Punt/Avui pour la périphérie (Catalogne). Le choix de ces quotidiens tient d'une part à leur caractère de «référence ». Leur influence sur le public et les autres médias font que leurs pages sont particulièrement convoitées par les acteurs en quête de visibilité sociale. Ils constituent donc des espaces publics de débat et, parlà même, de construction des identités. D'autre part, chacun d'entre eux possède un profil idéologico-politique particulier. Tandis qu'El País assume une ligne éditoriale de centre-gauche favorable au dialogue avec les différentes " nationalités ${ }^{5}$ » existant sur le territoire espagnol, El Mundo s'illustre par un discours conservateur, hostile à la décentralisation de l'État ${ }^{6}$. La Vanguardia peut être considérée comme l'expression d'un catalanisme politique modéré qui s'oppose à l'idéologie indépendantiste de gauche d'El Punt/Avui, le seul de quatre journaux à être édité en catalan. Compte-tenu de ces différences, il est possible de postuler que les journaux représentent le débat anti-taurin de manière plus ou moins partisane, en cela que les acteurs impliqués appartiennent à des communautés à la fois commune et différenciée (l'espagnole vs. la catalane ; la nationale $v$ s. la régionale) et que leurs récits s'ajustent à un public ayant des sensibilités politiques différentes.

5 Terme issu de l'article 2 de la Constitution de 1978.

6 Sur le profil idéologique des journaux, voir Thouverez (2010: 101-106) et Perales García (2014). 
Cette contribution aura pour but de vérifier si les journaux offrent une information conforme à leur ligne idéologique brièvement décrite ici et s'ils utilisent l'interdiction de la corrida pour réaffirmer leur propre discours identitaire. L'étude des macropropositions sémantiques - grandes unités de sens qui résument les différentes propositions formulées dans le texte (Van Dijk, 1990 : 81) - et de la caractérisation des acteurs permettra de déterminer si la représentation du conflit s'articule en fonction des règles du carré idéologique de Van Dijk (1990 : 121). C'est-à-dire, si les journaux accentuent les actions positives de leurs alliés tout en minimisant celles de leurs adversaires - et inversement. Une attention particulière sera également portée aux techniques de rhétorique employées par chaque quotidien pour faire accepter ses thèses, de même qu'aux déictiques, connecteurs logiques et nature des arguments employés dans les textes.

\section{Les médias, acteurs du clivage entre centre et périphérie}

\subsection{Presse nationale}

El Mundo adopte une position partisane dans le conflit. Sa conception du problème est clairement exprimée dans un éditorial du 27 juillet 2010 : «L'interdiction de la corrida est une question politique qui n'a rien à voir avec le droit des animaux. Ce que l'on veut [...] c'est continuer à rompre les liens culturels qui permettent d'identifier la Catalogne au reste de l'Espagne ${ }^{7}$ ». Le verbe "continuer à » présuppose l'existence d'une querelle identitaire ancienne. Selon El Mundo, celle-ci remonte à 1980, date de l'arrivée au pouvoir du parti nationaliste Convergència i Unió en Catalogne : «Depuis lors, on ne promeut que les émotions et les sentiments d'appartenance exclusivement vernaculaires. Les sentiments d'appartenance à l'Espagne, en revanche, sont amputés $^{8}$ ». La métaphore corporelle sous-entend que le nationalisme catalan n'aurait d'autre dessein que de démembrer le corps social, culturel et politique de l'Espagne. C'est pourquoi le quotidien conservateur s'insurge contre l'abolition de la «Fête nationale », au moyen de trois stratégies discursives.

La première entend démontrer que la corrida est une tradition culturelle commune à l'ensemble du territoire espagnol. De nombreux historiens soulignent ainsi l'ancrage social et la "glorieuse époque» de la tauromachie en Catalogne ${ }^{9}$. La deu-

7 «Bajonazo de los socialistas a una fiesta española», El Mundo, 27/7/2010, p. 3. Toutes les traductions sont des auteurs.

8 FERRER MOLINA V. «La vaquilla », El Mundo, 30/7/2010, p. 14.

9 FELICES R. «Una época gloriosa », El Mundo, 27/7/2010, p. 19. 
xième consiste à assimiler l'interdiction à une attaque contre "la Culture " et "l'Histoire », à travers de multiples références aux œuvres de García Lorca, Goya ou Hemingway. El Mundo discrédite enfin les partisans de l'abolition: tandis que les autorités catalanes sont accusées de "persécution » envers les aficionados, les députés ayant voté en faveur de l'abolition sont qualifiés de «fondamentalistes», «ignorants », « totalitaires », « culturicides » et « voyous » ${ }^{10}$.

Le journal de centre-gauche El País adopte un positionnement plus modéré et appelle au respect de la décision catalane : «L'initiative législative populaire a prospéré [...] parce qu'elle se fait l'écho d'une demande citoyenne qui a obtenu le soutien de la majorité des députés, après avoir suivi les procédés exigés par le cadre juridique en vigueur ${ }^{11}$ ». Si le quotidien attribue l'interdiction à une pression sociale, il considère qu'elle n'était pas nécessaire puisque la corrida était vouée à disparaître. "Agonie», " oubli », "mort» sont les substantifs les plus souvent employés pour décrire le secteur taurin en Catalogne.

Les stratégies discursives du journal visent à légitimer le processus législatif et à dénoncer la «pollution identitaire » du débat par les nationalistes catalans ou espagnols. Les articles d'information et d'opinion suivent la ligne éditoriale et évoquent l'évolution des sociétés vis-à-vis de la cause animale: «Les polémiques autour de la corrida, de la chasse au renard, du traitement des animaux dans les fermes ou laboratoires [...] ont ravivé une question que le monde occidental se pose depuis le XVIIIe siècle au moins: les animaux ont-ils des droits ${ }^{12}$ ?». Ils soulignent également qu'« aucune référence aux identités nationales, à la langue ou à l'idiosyncrasie des peuples ${ }^{13}$ " ne figure sur la pétition ayant recueilli 180.000 signatures et que « beaucoup de gens considèrent qu'organiser un spectacle fondé sur la maltraitance animale dépasse les frontières du civilisé ${ }^{14}$ ».

Contrairement à El Mundo, El País offre une pluralité d'opinions et de perspectives. Il existe pourtant certains parallélismes entre les couvertures des deux médias : mise en accusation de leurs adversaires respectifs ; recours à l'universel pour justifier leurs positions (atteinte contre la Culture et l'Histoire mondiales pour El Mundo, débat sur les droits des animaux au sein des sociétés occidentales pour El País), condamnation du nationalisme catalan et non reconnaissance du nationalisme espagnol qui semble affleurer dans bon nombre de leurs discours.

10 CAMINO P. « Un respecto a la afición », El Mundo, 28/7/2010, p. 6 ; DE ESTEBAN, J. « Hacia el totalitarismo », El Mundo, 29/7/2010, p. 7. SOTO VIÑOLO, J. «El puntillazo a la Fiesta en Cataluña», El Mundo, 25/7/2010, p. 57.

11 «De toros e identidades », El País, 29/7/2010, p. 20.

12 CortinA A. « ¿Tienen derecho los animales? 》, El País, 29/7/2010, p. 21.

13 Roger M. «Banderillas negras en Cataluña », El País, 25/7/2010, p. 49.

14 RAMONEDA J. «Crueldad e identidad», El País, 29/7/2010, p. 13. 


\subsection{Presse catalane}

Le journal favorable à la cause catalane, La Vanguardia, affiche une posture modérée qui condamne l'interdiction de la corrida, au motif qu'elle intervient dans un contexte tendu. Il est ainsi rappelé que des querelles ont éclaté à propos du statut d'autonomie et qu'il convient d'être attentif à la perception du vote catalan dans le reste de l'Espagne et à l'étranger : "L'interdiction de la corrida peut être irrémédiablement perçue comme une décision destinée à briser encore plus les liens émotionnels avec l'Espagne. Est-ce cela que veulent transmettre au monde les principales forces politiques catalanes? ${ }^{15}$ ». Le journal rejette les postures les plus radicalisées et défend la corrida au moyen d'arguments de valeur relatifs à son identité. Les combats de taureaux, affirme La Vanguardia, font aussi bien partie de la culture catalane qu'espagnole et il serait « farfelu » de les éradiquer. Le journal attribue aussi un caractère discriminatoire à la décision, puisqu'elle prive certains citoyens d'une forme de loisir. Malgré ses appels à l'apaisement, le quotidien n'hésite pas à dénoncer ses adversaires politiques, tels que la droite espagnole, qu'il accuse de «tomber dans la tentation du casticismo [une vision essentialiste de l'identité espagnole] ${ }^{16}$ ". Fidèle à une version modérée du catalanisme, qui veut que la Catalogne soit traitée de manière distinctive tout en jouant un rôle dans la modernisation de l'Espagne, La Vanguardia ajoute : «cette même discussion aura lieu dans quelques années à Madrid, quand de nouvelles générations arriveront à maturité ».

Le journal indépendantiste El Punt/Avui approuve l'abolition et estime qu'il ne s'agit pas « d'un débat identitaire, ni d'une intention politique de répliquer au coup porté au statut d'autonomie ${ }^{17}$ ", mais d'une question de défense de la cause animale. La suppression de la corrida est justifiée par le biais d'arguments de valeur tels que:les combats de taureaux sont " une diversion fondée sur la torture », une tradition " brutale et lamentable », un spectacle « indigne d'une société civilisée $»^{18}$. L'adjectif « civilisé » témoigne d'une incohérence discursive. En effet, s'il sous-entend que la Catalogne a atteint un plus grand degré de civilisation en abolissant une pratique estimée digne d'une société barbare, le journal adopte inévitablement un positionnement identitaire, en cela qu'il associe plusieurs valeurs et comportements à "son » groupe et à « l'autre » groupe. Cet exemple n'est pas isolé. L'affirmation suivante, « Il y a dix ans, le gouvernement canarien a approuvé une loi similaire, sans que personne n'y voit

15 «Catalunya y los toros», La Vanguardia, 25/7/2010, p. 26.

16 «Y ahora la tentación casticista», La Vanguardia, 29/7/2010, p. 16

17 «Catalunya, sense curses de toros a partir de $2012 »$, El Punt/Avui, 29/7/2010, p. 20.

18 "Catalunya se situa a un pas de l'abolició de les curses de braus », El Punt/Avui, 27/7/2010,

p. 1 ; ESPADA F. « Un país més digne», El Punt/Avui, 29/7/2010, p. 4; «La prohibició de les curses de braus té raons », El Punt/Avui, 27/7/2010, p. 4. 
l'intention de porter préjudice à la culture espagnole 19 ", semble indiquer que la tauromachie est perçue comme un apport culturel extérieur. Cette stratégie consistant à se distancier d'une culture considérée comme espagnole est intéressante dans la mesure où il serait possible d'objecter que la pratique de la corrida est attestée depuis le XIVe siècle en Catalogne et qu'elle semble donc antérieure à la construction de l'Étatnation espagnol. À ce propos, de nombreux défenseurs de l'identité catalane, comme les peintres Joan Miró et Salvador Dalí, le chanteur Joan Manuel Serrat ou le poète Pere Gimferrer, fréquentaient les arènes.

El Punt/Avui soutient également que l'abolition entraînera la reconnaissance de la singularité catalane à l'étranger. Un journaliste, qui salue l'événement par le titre «Adieu Espagne noire!», en référence à la légende selon laquelle l’Espagne serait un bastion d'intolérance, de fanatisme religieux et d'obscurantisme, estime que «l'on ne pourra plus jamais voir la Catalogne comme une estampe de la Carmen de Bizet ${ }^{20}{ }^{2}$. Notons que l'emploi de déictiques (eux/nous, ici/là-bas) renforce la dichotomie entre le centre et la périphérie et que le journal n'hésite pas à s'inclure dans les récits quand il affirme que "l'abolition renforce notre différenciation identitaire, culturelle, tout comme notre cause politique ${ }^{21}$ » ou que «la tauromachie était un spectacle qui nous dénigrait en tant que sociétée ${ }^{22}$ ». El Punt/Avui articule donc un discours nationaliste qui occulte l'existence de minorités culturelles et politiques en Catalogne.

\section{Conclusion : réaffirmation d'un discours basé sur la différence}

Les récits produits par les différents quotidiens s'articulent selon les principes du carré idéologique de Van Dijk: tous adoptent une ligne éditoriale similaire à celle de leurs alliés politiques et idéologiques. El Mundo, proche de la droite "espagnoliste", perçoit la décision du parlement catalan comme un défi à l'égard de la nation espagnole. Ses récits tendent à réaffirmer l'unité culturelle nationale et rejeter toute tentative de remise en question de celle-ci. Inversement, El País adopte une ligne modérée qui reflète le débat existant au sein de la gauche espagnole à l'égard de la tauromachie et, plus largement, de l'organisation politico-territoriale de l'Espagne. Bien qu'il soit contraire à toute forme d'abolition, El País appelle au respect du mouvement social anti-taurin. En revanche, linstrumentalisation du débat est critiquée dans la mesure où le déploiement d'idéologies nationalistes fragilise l'entente entre le centre et la pé-

\footnotetext{
19 Ibidem.

20 BRou L. "Adéu Espanya negra », El Punt/Avui, 29/7/2010, p. 14.

21 CABRÉ, J. «Després dels toros », El Punt/Avui, 31/7/2010, p. 17.

22 EsPaDA, F. «Un país més digne», El Punt/Avui, 29/7/2010, p. 4.
} 
riphérie. La Vanguardia rejoint la position d'El País et considère l'abolition comme une source de tensions inutiles. Le discours du journal "catalaniste » diffère néanmoins de son concurrent madrilène quand il souligne la modernité de la Catalogne vis-à-vis du reste de l'Espagne. Le quotidien indépendantiste El Punt/Avui estime enfin que l'abolition est positive, en cela qu'elle générera une plus grande cohésion au sein de la société catalane et une reconnaissance de sa singularité à l'extérieur.

Il apparaît donc que tous les quotidiens utilisent le conflit pour réaffirmer leur propre discours identitaire et accentuer les différences entre le groupe auquel ils s'identifient et celui auquel ils s'opposent. Cette différentiation s'effectue grâce à la généralisation ("L'Espagne/les Espagnols » vs. « la Catalogne/les Catalans ») et la simplification (les acteurs ayant des sentiments identitaires partagés sont absents des récits). Les relations entre le centre et la périphérie sont également décrites au moyen d'un lexique soulignant la désunion (" rupture », "brèche », "confrontation ", "liens brisés », etc.) et la portée de l'événement amplifiée par des métaphores issues du lexique tauromachique (" coup de grâce», «banderilles noires », «la fête en deuil»). L'usage de déictiques et d'attributs permet de forcer les traits distinctifs de chaque groupe: selon le point de vue adopté, les Catalans sont «civilisés», "modernes», «proches de l'Europe » vs. "intolérants » et " arrogants». Quant aux Espagnols, ils sont tantôt associés à la défense de la culture universelle, tantôt au barbarisme, au fanatisme et aux valeurs du passé.

En mettant en scène une réalité orientée vers la confrontation, les journaux prennent une part active au conflit. Le lecteur est donc invité à se positionner, à accepter ou rejeter les symboles de l'une ou de l'autre communauté et à soutenir leur projet politique respectif: hégémonie nationale, d'une part; revendication particulariste ou indépendantiste, d'autre part. Il faut toutefois reconnaître que le degré d'implication des journaux varie en fonction de leur idéologie et que la représentation du conflit est fortement influencée par le contexte et le caractère symbolique du taureau. Preuve en est que la décision de la Catalogne d'interdire la mise en scène d'animaux dans les cirques n’a suscité, en octobre 2013, aucune polémique en Espagne.

\section{Bibliographie}

BORRAT H. (1989) : El periódico, actor político. Barcelone : Gustavo Gili.

Charaudeau P. (2006) : «Identités sociales, identités culturelles et compétences ». In http://www.patrick-charaudeau.com/Identites-sociales-identites.html

MAíz R. (1997) : Nacionalismo, Democracia y Federalismo. Barcelone: Fundació Rafael Campalans.

Louw E. (2005) : The media and political process. Londres : Sage. 
Perales Garcia C. (2014) : Premsa i autodeterminació: Catalunya i Euskadi dins l'Espanya de la Transició. Barcelone : UOC.

Perez-Agote A. (1993): "Las paradojas de la nación». In Revista española de investigaciones sociológicas, $\mathrm{n}^{\circ} 61, \mathrm{pp} .7-22$.

Soriano Procas M. (2012): "El fin de los toros: del derecho animal al debate identitario », in CASTELLó E., ed. (2012): La mediatización del conflicto político. Barcelone : Laertes, pp.155-169.

VAN DIJK T. (1990) : La noticia como discurso. Comprensión, estructura y producción de la información. Barcelone : Paidós.

ThOUVERez L. (2010): Violence d'État et médias. Le traitement informatif du GAL dans la presse française et espagnole de référence. Paris : LGDJ.

WodAK, R., Meyer, M. (2003) : Métodos de análisis crítico del discurso. Barcelone : Gedisa. 